\title{
Chemosynthesis, characterizations and electrochromic properties of a novel solution-processable donor-acceptor type copolymer
}

\author{
Hui Luo ${ }^{1, a}$, Zhen $\mathrm{Xu}^{1, \mathrm{~b}}$, Jinsheng Zhao ${ }^{2, \mathrm{c}}$ and Weiyu Fan ${ }^{1, \mathrm{~d}}$ \\ ${ }^{1}$ State Key Laboratory of Heavy Oil Processing, China University of Petroleum (East China), \\ QingDao 266555, China \\ ${ }^{2}$ Shandong Key Laboratory of Chemical Energy Storage and Novel Cell Technology, Liaocheng \\ University, Liaocheng 252059, China \\ aluohuidcnh@163.com, bxz0215@126.com, cj.s.zhao@163.com, dfanwyu@upc.edu.cn
}

Keywords: Chemical copolymerization, Electrochromic properties, donor-acceptor type, Spectroelectrochemistry.

\begin{abstract}
Chemical copolymerization of 4, 7-bis (4-hexyl-5-trimethylstannyl-2-thieny l) -2,1,3-benzothiadiazole and 2, 5-didecyloxy-1,4-dibromobenzene was carried out in refluxed toluene with bis (triphenylphosphine) palladium(II) chloride $(\mathrm{Pd}(\mathrm{PPh} 3) 2 \mathrm{Cl} 2)$ as catalyst to give donoracceptor type poly (4 , 7 -bis (4-hexyl-2-thienyl) -2,1,3-benzothiadiazole-co-p-didecyloxybenzene) (PTBTB) via Stille-coupling reaction. Spectroelectrochemical and electrochromic properties of the resulting copolymer were characterized by cyclic voltammetry (CV) and UV-Vis-NIR spectroscopy. Cyclic voltammetry showed a definite electrochemical redox behavior of PTBTB polymer during the p-doping progress and an explicit reduction peak at about $-1.4 \mathrm{~V}$ at the n-doped state. And spectroelectrochemical studies demonstrated that the hybrid polymer film had distinct electrochromic properties with color changing from red in the reduced form $(0 \mathrm{~V})$ to light gray upon step-wise oxidation $(1.03 \mathrm{~V})$ and tile blue at the fully oxidized state $(1.4 \mathrm{~V})$. Further kinetic studies results indicated that the hybrid polymer had decent optical contrast ratios (34.3\% for $517 \mathrm{~nm}$ and $65.2 \%$ for $1600 \mathrm{~nm})$, favorable coloration efficiency $(238 \mathrm{~cm} 2 / \mathrm{C})$ and fast response time $(0.6 \mathrm{~s})$. The material hold promise for electrochromic applications.
\end{abstract}

\section{Introduction}

In recent years, electrochromic technique, especially due to broad application prospects of electrochromic devices, such as thesmart windows, color sunglasses, glare-free mirror, color display, electronic paper and military camouflage and other fields, have caused widespread concern at home and abroad [1]. A reversible optical/color change observed in a material because of the electrochemical redox reaction at different applied external voltages is defined as electrochromism. Organic conjugated conductive polymersas an important class of electrochromic materials have received much more attention because of its unique advantages including lower cost, better toughness, easier processing, richer color change and easier molecular structure modification to improve the electrochromic properties than the inorganic materials. In the family of conducting polymers, donor-acceptor type materials, which are composed of alternating electron-donating groups and electron-withdrawing groups, have been documented to be with high hole mobility capability and can optimize the spectral absorption range and the energy band-gap, thus presenting distinguished optical and electronic properties [2].

Benzothiadiazole (BTD) with electron-withdrawing imine nitrogens $(\mathrm{C}=\mathrm{N})$ has only recently been established as a good acceptor-type unit for D-A conjugated systems. Previously reported benzothiadiazole based D-A type polymers are usually obtained by coupling with electron donating thiophene or its derivatives as donor units. Well definedextension of the conjugated length in its structure between benzothiadiazole and thiophene groups are often associated with low band-gap [3], which can lead to specific optoelectronic properties [4,5].However, if we want to achieve the electrochromic films by large-scale production, the need for high solubility of the polymers is of greatest importance. 
Taking into consideration the demand for both low energy band-gap and improvedsolubility, we have succeeded in preparation of a new donor-acceptor copolymer with high molecular weights, excellent solubility and low optical band-gap at about $1.98 \mathrm{eV}$. In addition, the spectroelectrochemical and electrochromic properties of the resulting PTBTB copolymer film are detailedly described in this article.The polymer exhibits multichromic properties with three distinctive colors and significant changing in UV-Vis-NIR absorption spectra at different applied potentials.

\section{Experimental}

\subsection{General.}

All chemicals were obtained from Aldrich Chemical and directly used without any other purification.Polymer thin films were prepared by spray-coated onto ITO/glass working electrode for electrochemical measurements and optical studies. Cyclic voltammetry (CV) was performed using a $\mathrm{CHI} 760 \mathrm{C}$ workstation in $0.1 \mathrm{M} \mathrm{TBAHF}_{6} / \mathrm{ACN}$ at a scan rate of $100 \mathrm{mV} / \mathrm{s}$ with platinum wire as the counter electrode, and Ag wire as the pseudo-reference electrode (-0.03 V vs. SCE). UV-Vis-NIR spectra of the polymer film were recorded on a Varian Cary 5000 spectrophotometer at a scan rate of $600 \mathrm{~nm} / \mathrm{min}$.Elemental analyses were performed on a Carlo-Erba 1160 elemental analyzer. Waters 1515-2414 gel permeation chromatography (GPC) was used to determine the molecular weight of the polymer. Photographs of the polymer film were taken by using a Canon (Power Shot A3000) digital camera.

\subsection{Synthesis.}

1,4- Didecyloxybenzene (2) was prepared in high yield through alkylation of hydroquinone (1) in the presence of 1-bromine decane in DMF solution (yields, 91\%) and subsequent bromination of the obtained alkoxy benzene (2) with $\mathrm{HBr}$ in glacial acetic acid under reflux heat treatment for $8 \mathrm{~h}$ (yields, 82\%). The Stille coupling reaction of 2,5-didecyloxy-1,4-dibromobenzene with 4,7-bis(4-hexyl-5-trimethylstannyl-2-thienyl)-2,1,3-benzothiadiazole in the presence of $\mathrm{Pd}\left(\mathrm{PPh}_{3}\right)_{2} \mathrm{Cl}_{2}$ as catalyst, gave the crude polymer production, which was then purified by Soxhlet extraction with methanol and acetone to afford poly(4,7-bis(4-hexyl-2-thienyl)-2,1,3-benzothiadiazole-co-p-didecyloxybenzene) as red solid in 83\% yield. GPC for $\mathrm{C}_{52} \mathrm{H}_{76} \mathrm{~N}_{2} \mathrm{O}_{2} \mathrm{~S}_{3}: \mathrm{M}_{\mathrm{n}}: 20.3 \mathrm{kDa}$, PDI: 1.91. Elem. Anal. Calcd. for $\mathrm{C}_{52} \mathrm{H}_{76} \mathrm{~N}_{2} \mathrm{O}_{2} \mathrm{~S}_{3}: \mathrm{C}$, 72.85\%; H, 8.93\%; N, 3.27\%; O: 3.73\%; S, 11.22\%. Found: C, 72.41\%; H, 9.03\%; N, 3.32\%; O, $3.80 \%$; S, $11.44 \%$.
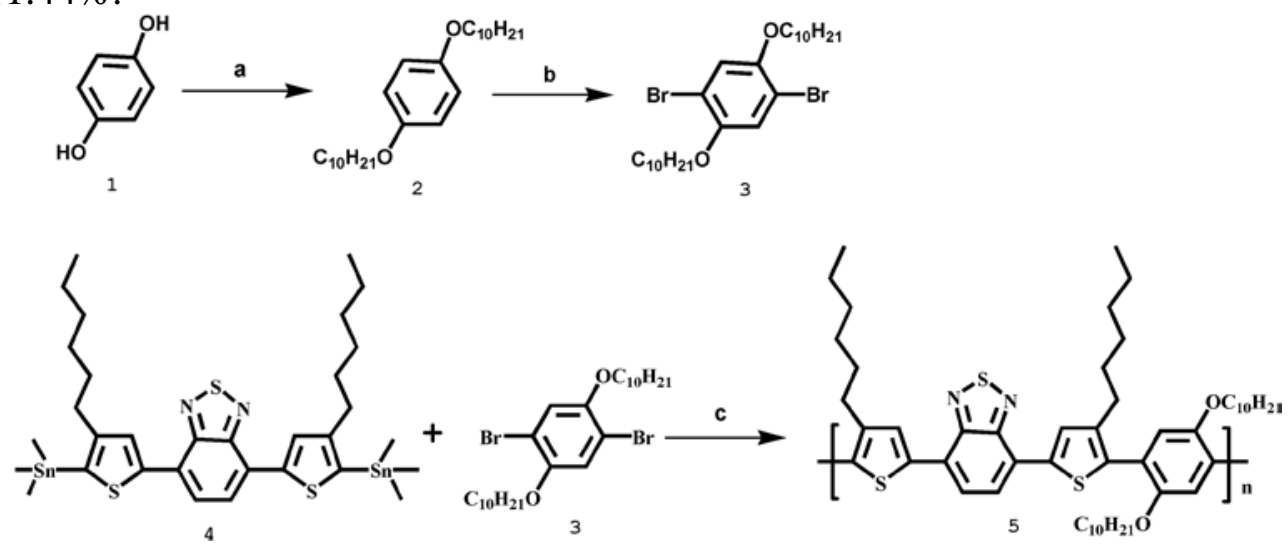

Scheme 1.Synthesis of the polymer.

\section{Results and discussion}

\subsection{Cyclic voltammetry.}

In order to investigate the electrochemical redox behavior of PTBTB,cyclic voltammetry (CV) studies were performed in $0.1 \mathrm{M} \mathrm{TBAHF}_{6} / \mathrm{ACN}$ solution. $\mathrm{CV}$ curve (figure 1 ) showed the distinct anodic redox behavior and cathodic reduction process. In addition, the ionization potentials $\left(\mathrm{E}_{\mathrm{HOMO}}\right)$ and the electron affinity ( $\mathrm{E}_{\mathrm{LUMO}}$ ) of were calculated according to the following empirical equations: 
$\mathrm{HOMO}=-\left(\mathrm{E}_{\text {ox,onset }}+4.4\right)$ and $\mathrm{LOMO}=-\left(\mathrm{E}_{\text {red,onset }}+4.4\right)$, where the $\mathrm{E}_{\text {ox,onset }}$ and $\mathrm{E}_{\text {red,onset }}$ are the onset oxidation potential and the onset reduction potential versus standard calomel electrode (SCE), respectively. And the electrochemical band-gap of were calculated to be $1.86 \mathrm{eV}$ by the subtraction of the HOMO level from the LOMO level. Other electrochemical data were all listed in Table 1 in detail.

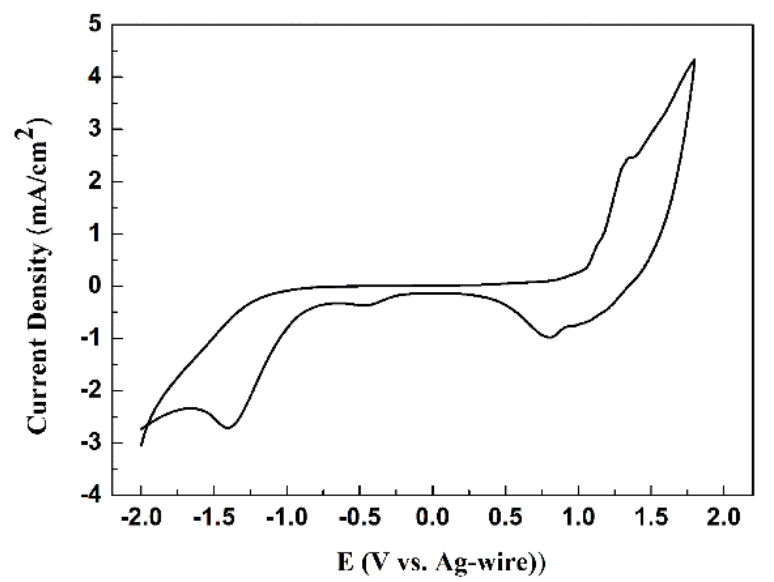

Figure 1. Cyclic voltammogram of PTBTB film casted on ITO/glass electrode in 0.1 TBAHF6/ACN at $100 \mathrm{mV} / \mathrm{s}$

Table 1 electrochemical and optical data of PTBTB

\begin{tabular}{cccccccc} 
Polymer & $\begin{array}{c}\mathrm{E}_{\text {ox,onset, }}, \text { vs.Ag } \\
(\mathrm{V})\end{array}$ & $\begin{array}{c}\mathrm{E}_{\text {red,onset, }} \\
(\mathrm{V})\end{array}$ & $\mathrm{E}_{\mathrm{g}, \mathrm{ec}}(\mathrm{eV})$ & $\begin{array}{c}\lambda_{\text {onset }} \\
(\mathrm{nm})\end{array}$ & $\mathrm{E}_{\mathrm{g}, \mathrm{op}}(\mathrm{eV})$ & $\mathrm{HOMO}(\mathrm{eV})$ & $\mathrm{LUMO}(\mathrm{eV})$ \\
\hline PTBTB & 1.02 & -0.84 & 1.86 & 627 & 1.98 & -5.39 & -3.53 \\
\hline
\end{tabular}

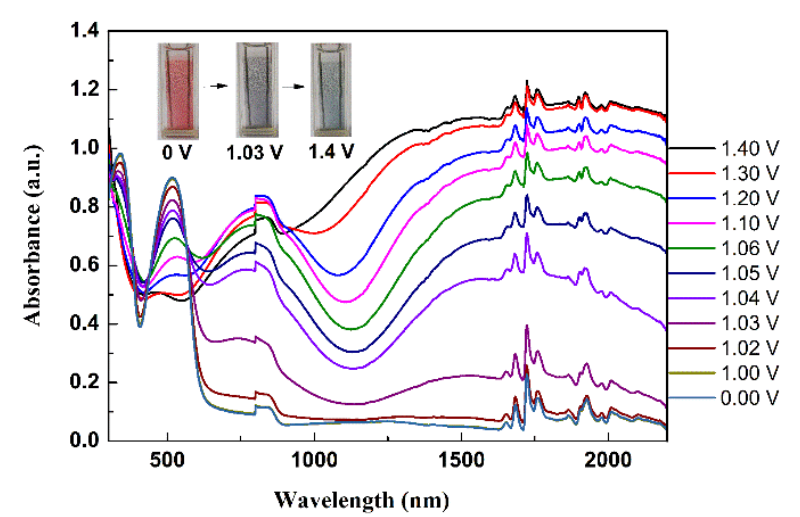

Figure 2. UV-Vis-NIR absorption spectra of PTBTB filmon ITO electrode atvarious applied potentials between $0 \mathrm{~V}$ and $1.40 \mathrm{~V}$.

\subsection{Spectroelectrochemistry.}

The absorption spectra of PTBTB in thin film at various applied potentials are performed and distinct absorption changes could be observed in Fig. 2. At the neutral state, PTBTB film exhibits two major characteristic absorption bands centered at 344 and $517 \mathrm{~nm}$, which can be assigned to the strong $\pi-\pi^{*}$ transition and the internal charge transfer (ICT) between the thiophene donor andbenzothiadiazole acceptor moiety, respectively. Opticalband-gap for this polymer in the film state was estimated from the lowest-energy band edge of the UV-Vis-NIR absorption spectra.Optical band gap of PTBTB was calculated to be $1.98 \mathrm{eV}$, which is significantly larger than the electrochemical band-gap. When the applied potential reached $+1.03 \mathrm{~V}$,the intensities of CT transition bands decreased and new absorption band at about $800 \mathrm{~nm}$ were detected due to the formation of charge 
carriers (polarons). Meanwhile, red color of the film at neutral state $(0 \mathrm{~V})$ turned into light gray at intermedia doped state $(+1.03 \mathrm{~V})$. Atfully oxidized state $(+1.4 \mathrm{~V})$, low-energy bipolaronic band at over $1200 \mathrm{~nm}$ in the near-infrared (NIR) region significantly increased. And the color of the film turned into tile blue finally. In addition, the changes in percentage transmittance $(\Delta T \%)$ between the neutral $(0 \mathrm{~V})$ and oxidized states $(1.4 \mathrm{~V})$ were found as $34.3 \%$ for $517 \mathrm{~nm}$ with a response time of 0.6 s. The coloration efficiency (CE) was calculated to be $238 \mathrm{~cm}^{2} / \mathrm{C}$. It is worth noting that optical contrastcan reach up to $65.2 \%$ in the NIR region for PTBTB film make this electrochromic material good candidate to be used in NIR applications [6].

\section{Conclusions}

A novel benzothiadiazole-based donor-acceptor type conjugated polymer has been designed and successfully synthesized via Stille-coupling reaction. The resulting copolymer has high solubility in some common organic solvents due to the incorporation of long alkoxy side chain.It is noted that PTBTB has red color in the neutral state and switches to light gray/tile blue upon oxidation. The electrochemical and optical band gaps of the polymer film are found to be $1.86 \mathrm{eV}$ and $1.98 \mathrm{eV}$, respectively. Moreover, good redox behavior, high CE and contrast ratio, and fast response time of this novel material make it excellent candidate for electrochromic application.

\section{Acknowledgements}

The work was financially supported by the Postgraduate Innovation Project of China University of Petroleum (East China) (YCX2015022) and the Fundamental Research Funds for the Central Universities (15CX06049A).

\section{References}

[1] H. H. Wang, M. M. Yan, Z. Y. Jiang. Electrochromic properties of rhodium oxide films prepared by a sol-gel method. Thin Solid Films. 401 (2001) 1, 211-215.

[2] Z. G. Zhang, J. Z. Wang. Structures and Properties of Conjugated Donor-Acceptor Copolymers for Solar Cell Applications. Journal of Materials Chemistry. 22 (2012) 10, 4178-4187.

[3] I. Kmínek, D. Výprachtický, J. Kř́žž, et al. Low-Band Gap Copolymers Containing Thienothiadiazole Units: Synthesis, Optical, and Electrochemical Properties. Journal of Polymer Science: Part A: Polymer Chemistry. 48 (2010) 13, 2743-2756.

[4] P. M. Beaujuge, S. Ellinger, J. R. Reynolds. Spray Processable Green to Highly Transmissive Electrochromics via Chemically Polymerizable Donor-Acceptor Heterocyclic Pentamers. Advanced Materials. 20 (2008) 14, 2772-2776.

[5] A. Durmus, G. E. Gunbas, L. Toppare, et al. A neutral state green polymer with a superior transmissive light blue oxidized state. Chemical Communications. (2007) 31, 3246-3248.

[6] A. M. McDonagh, S. R. Bayly, D. J. Rile, et al. A Variable Optical Attenuator Operating in the Near-Infrared Region Based on an Electrochromic Molybdenum Complex. Chemistry of Materials, 12 (2000) 9, 2523-2524. 